\title{
Terrains et destins de Maurice Leenhardt de Michel Naepels et Christine Salomon (éds)
}

Isabelle Leblic

\section{(2) OpenEdition \\ 1 Journals}

Édition électronique

URL : http://journals.openedition.org/jso/4732

DOI : $10.4000 /$ jso.4732

ISSN : $1760-7256$

Éditeur

Société des océanistes

\section{Édition imprimée}

Date de publication : 15 décembre 2008

Pagination : 348-350

ISBN : 978-2-85430-012-3

ISSN : 0300-953x

\section{Référence électronique}

Isabelle Leblic, "Terrains et destins de Maurice Leenhardt de Michel Naepels et Christine Salomon (éds) », Journal de la Société des Océanistes [En ligne], 126-127 | Année 2008, mis en ligne le 01 décembre 2008, consulté le 24 septembre 2020. URL : http://journals.openedition.org/jso/4732 ; DOI : https://doi.org/10.4000/jso.4732

Ce document a été généré automatiquement le 24 septembre 2020.

(c) Tous droits réservés 


\title{
Terrains et destins de Maurice Leenhardt de Michel Naepels et Christine Salomon (éds)
}

\author{
Isabelle Leblic
}

\section{RÉFÉRENCE}

Michel NAEPELS et Christine SALOMON (éds), 2007. Terrains et destins de Maurice Leenhardt, Paris, éditions de l'École des hautes études en sciences sociales, coll. Cahiers de l'homme, 165 p. \& 8 planches photos, annexe.

1 Cet ouvrage, qui rassemble six auteurs, avec une présentation générale et six articles, revenant sur l'œuvre et la personne de Maurice Leenhardt (1878-1954), missionnaire, élève de Marcel Mauss, proche de Lucien Lévy-Bruhl et " pionnier » de l'ethnologie kanak, se veut aussi un éclairage sur les conditions de production du savoir ethnographique ${ }^{1}$. S'inscrivant dans le « débat sur les rapports entre anthropologie et mission", ces auteurs, dans leurs participations, veulent ainsi éclairer l'œuvre scientifique du missionnaire, grâce à une critique des sources et à une mise en contexte, notamment la relation entre colonisation, mission et problématisation anthropologique. Il s'interroge aussi sur la nature de l'engagement de Maurice Leenhardt sur le terrain calédonien, qui oscille entre un réformisme colonial d'inspiration humaniste - qui engagea le missionnaire dans la défense des Kanak à de nombreuses reprises- et une phénoménologie religieuse avec l'étude de la mentalité kanak pour mieux la convertir...

2 Tout d'abord, les éditeurs scientifiques de l'ouvrage, Michel Naepels et Christine Salomon, reviennent sur ce va-et-vient «entre ethnologie et spéculations morales » dans leur présentation (pp.9-23). Puis viennent les six articles, répartis en deux parties : I. Colonisation et mission dans les années 1930 avec Benoît de L'Estoile (Une politique de l'âme: ethnologie et humanisme colonial, pp.27-49), Frédéric Keck 
(Mentalité mythique ou mystique? Leenhardt et Lévy-Bruhl, pp.51-67) et Michel Naepels (Notion de personne et dynamique missionnaire, pp. 69-91); II. Du réformisme colonial à l'indépendance kanak avec, à nouveau, Michel Naepels (Les pratiques ethnographiques de Maurice Leenhardt, pp. 95-115), Marie Pineau-Salaün (Pédagogie missionnaire, pédagogie coloniale, pp. 117-137) et Hamid Mokaddem (D’un usage kanak des sciences sociales : Jean-Marie Tjibaou lecteur de Maurice Leenhardt, pp. 139-157), pour se terminer sur un cahier (non paginé) de huit photos extraites de l'Album 1938-1939, Maurice et Jeanne Leenhardt (fonds 2 Num 13 des archives de NouvelleCalédonie) et une annexe (pp.161-165), la retranscription de l'intervention de JeanMarie Tjibaou au colloque Maurice Leenhardt (13 avril 1978, musée de l'Homme). Cet ouvrage assemble ainsi les contributions de trois spécialistes de la Nouvelle-Calédonie kanak en divers disciplines (anthropologie, sciences de l'éducation et philosophie) et de deux autres chercheurs : Frédéric Kerk, enseignant la philosophie à l'université Lille III et au Centre international de la philosophie française contemporaine de l'École normale supérieure, qui s'intéresse aux croisements entre la philosophie et l'anthropologie, notamment dans une dimension historique, à travers une généalogie de l'anthropologie française (de Comte à Lévi-Strauss, en passant par Lévy-Bruhl) et Benoît de L'Estoile, professeur agrégé d'anthropologie au département de sciences sociales de l'École normale supérieure, membre de l'IIIS'²-EHESS comme Michel Naepels et Marie Salaün. À ce propos, on peut regretter le manque de présentation et de rattachement institutionnel des contributeurs de ce volume. Rentrons quelque peu dans le détail des chapitres.

Dans leur présentation, Christine Salomon et Michel Naepels reviennent sur le pourquoi de cet ouvrage après celui, déjà ancien, de James Clifford (paru en français en 1987 [édition anglaise de 1982] sous le titre Maurice Leenhardt. Personne et mythe en Nouvelle-Calédonie, Paris, J.-M. Place). Il leur fallait en effet reprendre "les questions centrales des relations de Maurice Leenhardt avec le colonialisme, de l'individualisme et de la personne "dividuelle" - définie par l'ensemble de ses relations - [qui] ont été soulevées» (p.9) sur le forum de discussion Asaonet depuis 2003, afin d'expliquer "l'ampleur de son destin » dans deux univers, «le monde universitaire anglo-saxon » et la Nouvelle-Calédonie, via "les institutions scolaires et religieuses protestantes » bien sûr mais aussi « une partie du mouvement nationaliste kanak» (p. 9), sans oublier des figures aussi diverses que Jacques Lacan, Vincent Crapanzano, Marylin Strathern ou Philippe Descola qui le citent dans diverses publications, certaines des plus récentes (2005). Dans cette présentation, en suivant la construction de l'ouvrage, ces deux auteurs reviennent d'abord sur le réformisme colonial du pasteur Maurice Leenhardt (qui y passa plus d'une vingtaine d'années en plusieurs séjours s'étalant de 1902 à 1948), sans être pour cela un précurseur de l'anticolonialisme (p. 13), ce que développeront de L'Estoile et Kerk dans leurs contributions. Puis, ils exposent en quoi le destin remarquable de Leenhardt, « en rupture avec la figure malinowskienne de l'autorité en anthropologie » (p.14) est lié davantage à son statut de missionnaire. Pour sa part, Naepels revient plus en détail sur les pratiques ethnographiques du missionnaire, en s'intéressant notamment à la notion de personne dans son œuvre. C'est par la réalisation de son œuvre missionnaire (plus que scientifique) que Leenhardt, dans « le souci de promouvoir la morale chrétienne » fait de l'école, du mariage, etc. « une cible importante des changements envisagés [par le pasteur] en Nouvelle-Calédonie » (p. 17) : 
«Ses premiers textes missionnaires dans lesquels il dénonçait l'oppression des femmes, les mariages "forcés" ou arrangés dès la naissance, la polygynie et la dot auxquels il opposait le modèle du mariage monogame contracté librement entre jeunes gens protestants dans le but de fonder une famille nombreuse - en témoignent » (p. 17) les grandes monographies du pasteur-ethnologue occultent complètement ces faits uniquement mentionnés dans les écrits de la première période et donc souvent méconnus.

Enfin, ils terminent leur présentation en revenant sur les lecteurs actuels de Leenhardt, de Jean-Marie Tjibaou dans une politique de revalorisation de la culture kanak (voir Mokaddem dans ce volume) à James clifford qui réfléchit ainsi à l'écriture anthropologique hors du modèle malinowskien... (p. 19).

« En n'hésitant pas à analyser ses raccourcis ou ses erreurs, peut-être pouvons-nous retrouver aujourd'hui - grâce à ses publications et à ses archives - la parole d'un certain nombre de ses interlocuteurs kanak du début du Xx ${ }^{e}$ siècle. » (p. 19)

Il est en effet couramment admis aujourd'hui que Maurice Leenhardt a repris en grande partie à son compte les "écrivains kanak» qui lui fournirent de nombreux cahiers, bases de ses écrits, sans toujours les nommer.

7 Je ne reviendrai ici que sur les deux contributions de Michel Naepels, dans lesquelles il nous explique d'abord comment la notion de personne kanak vue par Leenhardt est grandement influencée par sa pratique missionnaire (pp.69-91), tout comme l'a été sa pratique ethnographique de terrain en Nouvelle-Calédonie (pp.95-113). La notion de personne « kanak » présentée par Leenhardt vient en contrepoint de celle exposée par Marcel Mauss : si ce dernier en avait proposé une définition avec une multiplicité de sens (philologique, social, juridique, théologique, cognitive et morale, sans faire ni linguistique ni psychologie, "Leenhardt fait et dit précisément l'inverse : il fit de la psychologie et de la linguistique, en déduisant l'expérience subjective des structures linguistiques, et il refusa l'évidence maussienne de l'universalité du sens interne de soi» (p. 70). Naepels montre bien comment l'«ethnolinguistique complexe et contestable [de Leenhardt] est étroitement liée à sa position de missionnaire » et qu'il s'appuie sur des interprétations linguistiques où « il accorde un poids déterminant aux structures linguistiques comme reflets des “mentalités" » (p. 71) ; comment également les erreurs de traduction ou d'interprétation des discours kanak en ajië (langue de la région de Houaïlou où il fut missionnaire) lui permettent de justifier ses propos. Il en déduit ainsi l'absence de conscience de soi, lacune qui justifie l'entreprise coloniale en général et la pratique missionnaire en particulier, pour faire passer les primitifs à l'état de civilisés!

8 Naepels revient aussi sur les notions de possession (pp.78-79). Bien qu'il note qu' " aucun des arguments proposés par Leenhardt à l'appui de ces thèses ne résiste à l'analyse linguistique » (p. 84), on peut regretter qu'il ne les présente pas plus en détail. Ainsi, le lecteur aurait été intéressé d'en savoir plus sur la question de la possession inaliénable/aliénable en liaison avec la notion de personne et qui a été reprise depuis Leenhardt par plusieurs auteurs, dont Françoise Ozanne-Rivierre, et dont il aurait pu tirer des enseignements.

9 L'analyse de Leenhardt de «l'enfermement social et cognitif des Kanak » (p. 85), bien que totalement erronée, correspondait toutefois aux idées de l'époque répandues dans 
tout l'univers colonial, et sur lesquelles reposent encore parfois aujourd'hui cette idée du colonisé qu'on ne dit plus "sauvage » mais « enfant » qu'il faut faire passer à l'état d'adulte (autrefois de civilisé) en le développant.

Dans son deuxième article, Michel Naepels revient sur les pratiques ethnographiques du missionnaire "pour comprendre quelle fut la source empirique de son savoir" (p. 95) et « reconstruire la façon dont Leenhardt travaillait, pour comprendre comment s'est élaborée son œuvre scientifique » (p. 96). Il montre bien comment la grande idée de Maurice Leenhardt fut de faire écrire par ses élèves kanak des cahiers qui lui permirent d'obtenir la plupart des matériaux de ces écrits scientifiques. À partir d'une analyse de sa correspondance à sa famille et de la reprise des cahiers et de leur traduction, Michel Naepels montre comment le missionnaire a pu construire son œuvre. Tout d'abord, c'est la traduction de la Bible en ajië qui lui a permis d'écrire le Vocabulaire de la langue de Houailou qui recèle de commentaires tout à fait intéressants au point de vue ethnographique qui n'ont pas toujours été repris dans ses autres écrits plus généraux, comme par exemple ce qui a trait à l'anthropophagie et aux violences faites aux femmes.Et le principal de son travail d'ordre ethnographique ne put être réalisé que lors de son troisième séjour (fin 1923 - début 1926). Il nous montre aussi mais c'est là un fait déjà bien connu de tous ceux qui s'intéressent à la NouvelleCalédonie et aux œuvres de Leenhardt - que ce sont les cahiers de ses élèves "Leenhardt encourageait toutes sortes de gens à écrire, sur des cahiers d'écolier, les légendes traditionnelles, les discours rituels, les chants qu'ils connaissaient bien » dixit James Clifford (1987 : 145) - qui lui permirent la rédaction des Documents (p. 105) dont ils sont tous co-auteurs. Et les Notes d'ethnologie néo-calédonienne trouvèrent leurs sources dans les cahiers de Bwesou Ërijiyi dont des chapitres entiers en furent la traduction. Naepels nous montre comment le «contexte de production » missionnaire « disparait de l'œuvre scientifique » (p. 109), ce qui conduit «à une ethnolinguistique discutable» (p. 113).

11 Je ne reviendrai pas plus avant sur les autres contributions que je laisse le soin aux lecteurs de découvrir par eux-mêmes.En conclusion, je dirais seulement que c'est un livre à lire, utile à plus d'un titre pour la connaissance de la Nouvelle-Calédonie.

\section{NOTES}

1. Les deux éditeurs scientifiques de cet ouvrage avaient organisé en 2001-2002 un séminaire sur le même thème à l'École normale supérieure. À part Hamid Mokaddem, les auteurs présents ici avaient exposé leur propos dans ce cadre. Cela aurait pu être indiqué dans la présentation de l'ouvrage.

2. Institut de recherche interdisciplinaire sur les enjeux sociaux (sciences sociales, politique, santé). 


\section{AUTEURS}

ISABELLE LEBLIC

CNRS LACITO, Villejuif 